\title{
A PREVALÊNCIA DA ADESÃO À VACINA CONTRA INFLUENZA: UMA REVISÃO DE LITERATURA
}

\author{
the PREVALENCE OF ADHESION TO VACCINE AGAINST \\ INFLUENZA: A LITERATURE REVIEW
}

\author{
Ana Raquel Silva Souza ${ }^{1}$ \\ Liliane de Almeida Cardoso ${ }^{2}$ \\ Kevin Fontelles Morais ${ }^{3}$
}

RESUMO: INTRODUÇÃO: o vírus da influenza caracteriza-se como uma patologia aguda e altamente infecciosa que atinge o sistema respiratório, podendo provocar pequenos surtos até graves epidemias. Atualmente, a forma mais eficaz de prevenção contra o vírus da Influenza e de suas complicações é feita por meio da imunização. OBJETIVO: identificar a prevalência da adesão à vacina contra influenza. MÉTODOS: trata-se de uma revisão integrativa de literatura no qual foram incluídos artigos publicados entre 2015 e 2019, nos idiomas português e inglês, a busca de dados foi realizada nas bases: BVS, SciELO, LILACS e PubMed, a partir da utilização dos descritores: "Imunização", "Vírus da Influenza A" e "Vacinas contra Influenza", com um total de 9 documentos científicos analisados no estudo. RESULTADOS: ainda há um desconhecimento da efetividade da vacina contra a influenza entre a população. Não obstante foi identificado que idosos, crianças, gestantes e pessoas com alguma comorbidade possuem um risco maior de desenvolver complicações devido à influenza. Apesar de a principal forma de prevenção ser a imunização anual, pode-se observar que há uma resistência e até mesmo o desconhecimento de estudantes e profissionais da saúde acerca do processo. CONCLUSÃO: portanto conclui-se que a forma mais eficaz de evitar a infecção influenza é por meio da imunização, que assume grande relevância na saúde pública do Brasil. Também é importante destacar que, a maioria dos estudos são voltados para os idosos, o que confirma a necessidade de que sejam desenvolvidos novos estudos que abranjam os demais grupos subsidiados pela rede pública de saúde.

\footnotetext{
1 Discente do curso de Enfermagem pela Universidade Estadual da Paraíba (UEPB), E-mail: anaraquel.coracao2@gmail.com.

2 Discente do curso de Enfermagem pela Universidade Estadual da Paraíba (UEPB), E-mail: almeida.lilianne@gmail.com.

${ }_{3}^{3}$ Discente do curso de Enfermagem pela Universidade Federal de Campina Grande (UFCG), E-mail: kevinfontellesuf@gmail.com.
} 
Palavras chave: Imunização; Vírus da Influenza A; Vacinas contra Influenza.

ABSTRACT: INTRODUCTION: influenza virus is characterized as an acute and highly infectious pathology that affects the respiratory system and can cause small outbreaks and even serious epidemics. Currently, the most effective way to prevent influenza viruses and their complications is through immunization. OBJECTIVE: to identify the prevalence of adherence to influenza vaccine. METHODOLOGY: this is an integrative literature review, which included articles published between 2015 and 2019 in the Portuguese and English languages. Data were searched in the following databases: BVS, SciELO, LILACS and PubMed, using descriptors: "Immunization", "Influenza A Virus" and "Influenza Vaccines", with a total of 9 scientific documents analyzed in the study. RESULTS: there is still a lack of knowledge about the effectiveness of influenza vaccine among the population. However, it was identified that the elderly, children, pregnant women and people with some comorbidity have a higher risk of developing complications due to influenza. Although the main form of prevention is annual immunization, it can be observed that there is resistance and even lack of knowledge from students and health professionals about the process. CONCLUSION: therefore, it can be concluded that the most effective way to prevent influenza infection is through immunization, which is of great relevance to public health in Brazil. It is also important to highlight that most studies are aimed at the elderly, which confirms the need to develop new studies that cover other groups subsidized by the public health network.

Keywords: Immunization. Influenza viruses A. Influenza vaccines. 


\section{INTRODUÇÃO}

O vírus da influenza se caracteriza como uma patologia aguda e altamente infecciosa que atinge o sistema respiratório, podendo provocar pequenos surtos até graves epidemias. Considera-se que cerca de 600 milhões de pessoas tenham ao menos um evento de influenza por ano. Além disso, indica-se que de toda a população brasileira, em torno de 22 mil pessoas morrem em virtude da influenza em tempos de epidemia. (MOURA, 2015; NEVES, 2016).

Segundo a Organização Mundial da Saúde (OMS, 2017), estima-se que a influenza acometa cerca de $5 \%$ a $10 \%$ dos adultos e de $20 \%$ a $30 \%$ das crianças, causando 3 a 5 milhões de casos graves e 250 mil a 500 mil mortes todos os anos. A doença é de elevada transmissibilidade e distribuição global, com tendência a se disseminar facilmente em epidemias sazonais e potencialmente causar pandemias.

A infecção pelo vírus Influenza pode frequentemente causar complicações, sendo a pneumonia a principal complicação relacionada à casos de hospitalizações e até o óbito. Não obstante, a infecção pelo vírus ainda pode ocasionar a exacerbação de doenças crônicas como a doença pulmonar obstrutiva crônica (DPOC), cardiopatias, dentre outras (BACURAU, 2018).

Atualmente, a forma mais eficaz de prevenção contra o vírus da Influenza e de suas complicações é feita por meio da imunização, onde no Brasil foi estabelecida por meio de campanhas, tendo início no ano de 1999 e repercutindo de forma positiva na redução de complicações, hospitalizações e gastos excessivos com medicamentos e mortes evitáveis até então (BACURAU, 2018).

Dentre os grupos prioritários recomendados pelo Ministério da Saúde para a vacinação anual contra o vírus da Influenza destaca-se os idosos com idade maior ou igual a 60 anos, indivíduos com doenças crônicas não transmissíveis (DCNT) e pessoas que convivem ou cuidam destas pessoas. Ainda, inclui-se crianças menores de cinco anos, uma vez que apresentam elevadas taxas de hospitalização e complicações respiratórias (BRASIL, 2017). 
Estudos demonstram que o crescimento da população idosa tem provocado uma mudança no perfil epidemiológico mundial, promovendo um novo padrão de morbimortalidade na população, caracterizada principalmente por doenças respiratórias, dentre elas, a gripe (BACURAU, 2019).

Porém, o Ministério da Saúde (BRASIL, 2017) destaca que apesar da existência de mecanismos de prevenção contra a doença e seus agravos, ainda há a resistência de uma determinada parcela da população como o grupo populacional formado por adultos e idosos não vacinados, que acabam por sofrer com complicações e óbitos, principalmente entre a população idosa.

Desinformação, disseminação de informações incorretas e equivocadas, valores religiosos e filosóficos, bem como a ausência de confiança nas instituições que produzem, fornecem e manipulam as vacinas são motivações para o movimento anti-vacinas, tal como a incerteza e adiamento para a adesão de vacinas. Esse contexto põe em risco não apenas a saúde do sujeito não vacinado, mas a de todos que lhe circundam (MIZUTA, 2019).

Ainda, estudos sobre coberturas vacinais e adultos e idosos portadores de doenças respiratórias pulmonares crônicas revelam que a prevalência de vacinação nesses grupos ainda é pouco dimensionada e que, apesar da recomendação, é observado uma taxa diminuta de adesão à vacinação (NEVES, 2016).

A “indecisão vacinal”, conforme a Organização Mundial de Saúde (OMS), é definida como o retardo na aceitação ou rejeição da vacinação relacionada à disponibilidade de serviços de vacinas. Mizuta (2019) observa que indivíduos que atrasam a aceitação ou renegam vacinam têm condutas complexas e precisam de monitoramento constante.

Considerando que a vacinação é forma mais eficiente de prevenir a influenza e que a mesma se configura como uma peça-chave em questões de saúde preventiva no que diz respeito ao fornecimento de assistência à saúde da população e a necessidade de discutir a temática em questão, este estudo tem como objetivo identificar a prevalência da adesão à vacina contra influenza no Brasil. 


\section{METODOLOGIA}

Trata-se de uma revisão integrativa de literatura (RIL) de caráter qualitativo, o qual tem a finalidade de sintetizar os estudos que apresentem maior relevância para temática em questão. Foi realizada a busca controlada de dados secundários em artigos científicos na Biblioteca Virtual de Saúde (BVS) e indexados nas bases de dados: Scientific Eletronic Library Online (SciELO), Literatura Latino-americana e do Caribe em Ciências da Saúde (LILACS); National Library of Medicine (PubMed), isto a partir da utilização dos descritores em saúde: "Imunização", "Vírus da Influenza A"; "Vacinas contra Influenza".

A busca dos dados foi conduzida com foco na seguinte questão norteadora "qual a prevalência da adesão à vacina contra o vírus da influenza A no Brasil?". Para triagem dos artigos utilizou-se como critérios de inclusão: resultados publicados entre 2015 e 2019, idiomas português e inglês; bem como materiais complementares disponíveis online e como critérios de exclusão: resultados publicados anteriormente ao ano de 2015 e estudos de literatura cinzenta. Com a aplicação de filtros e posteriormente a seleção de artigos, obteve-se um total de 9 documentos científicos analisados no estudo.

A coleta das informações ocorreu entre os meses de dezembro de 2019 e janeiro de 2020, mediante uso de formulário estruturado, adaptado (URSI, 2005) para as variáveis necessária à questão norteadora, apresentando-se conforme Quadro 1: identificação das publicações, características metodológicas dos estudos e conclusões.

A apresentação dos resultados e discussão dos dados obtidos foi feita de forma descritiva, possibilitando ao leitor a avaliação da aplicabilidade da revisão integrativa elaborada, de forma que fosse possível fornecer subsídios ao profissional da saúde em sua tomada de decisão cotidiana. 


\section{RESULTADOS E DISCUSSÃO}

\section{Os resultados da RIL foram organizados de acordo com os componentes} estruturantes dos artigos, de forma a delinear com clareza as variáveis de interesse.

\begin{tabular}{|c|c|c|c|}
\hline $\begin{array}{l}\text { TíTULO DO } \\
\text { ARTIGO }\end{array}$ & AUTORES & OBJETIVO & CONCLUSÃO \\
\hline 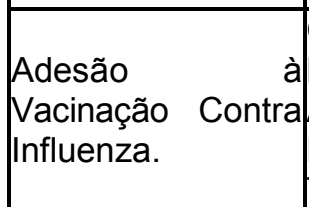 & $\begin{array}{l}\text { COSTA, } \\
\text { MENESES, N. F. } \\
\text { A; ANDRADE, P. } \\
\text { R; HINO, P; } \\
\text { TAMINATO, M. }\end{array}$ & \begin{tabular}{|l} 
Verificar a adesão a \\
uma campanha \\
vacinação contra \\
influenza.
\end{tabular} & \begin{tabular}{|l|} 
Percebeu-se que o enfermeiro tem pape| \\
essencial nas ações de promoção da \\
vacinação nos ambientes de educação \\
infantil visando ao aumento da cobertura \\
vacinal e à prevenção de agravos.
\end{tabular} \\
\hline & & & $\begin{array}{l}\text { É necessário incentivar a vacinação de } \\
\text { idosos com }\end{array}$ \\
\hline $\begin{array}{|lr|}\text { Fatores } & \\
\text { associados } & \text { à } \\
\text { adesão } & \text { à } \\
\text { vacinação } & \text { anti- } \\
\text { influenza } & \text { em } \\
\text { idosos } & \text { não } \\
\text { institucionalizados. } \\
\end{array}$ & $\begin{array}{l}\text { MOURA, R. F; } \\
\text { ANDRADE, F. B; } \\
\text { DUARTE, Y. Y. } \\
\text { A.O; LEBRÃO, } \\
\text { M. L; ANTUNES, } \\
\text { J. L. F. }\end{array}$ & $\mid \begin{array}{l}\text { Estimar a cobertura } \\
\text { vacinal contra } \\
\text { influenza em idosos e e } \\
\text { identificar os fatores } \\
\text { associados à adesão à } \\
\text { vacinação. }\end{array}$ & $\begin{array}{l}\text { menos de } 70 \text { anos e sem doenças } \\
\text { crônicas, assim como orientar os } \\
\text { profissionais de saúde para ampliar a } \\
\text { cobertura nos grupos com menor } \\
\text { participação nas campanhas. }\end{array}$ \\
\hline $\begin{array}{l}\text { Mortalidade por } \\
\text { doenças } \\
\text { cerebrovasculares } \\
\text { em idosos e a } \\
\text { vacinação contra a } \\
\text { influenza: Estado } \\
\text { de São Paulo, } \\
\text { Brasil, 1980-2012. }\end{array}$ & $\begin{array}{l}\text { BACURAU, A. A. } \\
\text { G. M; FERRA, } \\
\text { R. } \\
\text { DONALISIO, M. } \\
\text { R; FRANCISCO, } \\
\text { P. M. S. B. }\end{array}$ & 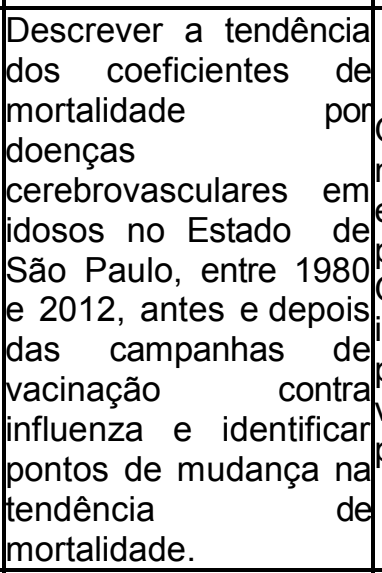 & \begin{tabular}{|l|} 
Os resultados mostraram redução da \\
mortalidade por DCbV no período \\
estudado, com diferentes variações \\
percentuais de queda dos coeficientes. \\
Os achados deste estudo adicionam \\
informações para o debate sobre o \\
possível efeito das campanhas de \\
vacinação na redução da mortalidade \\
por DCbV na população idosa.
\end{tabular} \\
\hline $\begin{array}{|lr|}\text { Prevalência } & \text { de } \\
\text { vacinação } & \text { contra } \\
\text { gripe } & \text { nas } \\
\text { populações } & \text { adulta } \\
\text { e } \quad \text { idosa } & \text { com } \\
\text { doença } & \\
\text { respiratória } & \\
\text { pulmonar crônica. }\end{array}$ & $\begin{array}{l}\text { BACURAU, } \\
\text { G. } \\
\text { FRANCISCO, } \\
\text { M. S. B. }\end{array}$ & $\begin{array}{l}\text { Estimar a prevalência } \\
\text { de vacinação contra } \\
\text { gripe nas } \\
\text { adulta e e e idosulações } \\
\text { doença com } \\
\text { pulmonar respiratória } \\
\text { (DRPC). }\end{array}$ & $\begin{array}{l}\text { Tanto os portadores de doença pulmonar } \\
\text { quanto os idosos em geral ( } \geq 60 \text { anos) } \\
\text { fazem parte de grupos prioritários para a } \\
\text { vacinação contra gripe tendo as } \\
\text { prevalências abaixo da meta estabelecida } \\
\text { pelo Ministério da Saúde. A } \\
\text { recomendação da vacina pelos } \\
\text { profissionais de saúde pode contribuir } \\
\text { para uma maior adesão desse grupo à } \\
\text { vacinação. }\end{array}$ \\
\hline $\begin{array}{|lr|}\text { Vacinação } & \text { contra } \\
\text { influenza } & \text { em } \\
\text { idosos de } & \text { Pelotas- } \\
\text { RS, } \quad 2014: & \text { um }\end{array}$ & $\begin{array}{l}\text { NEVES, R. G; } \\
\text { DURO, S. M. S; } \\
\text { TOMASI, L. }\end{array}$ & $\begin{array}{l}\text { Descrever } \\
\text { prevalência } \\
\text { vacinados } \\
\text { influenza e } \\
\end{array}$ & $\begin{array}{l}\text { A cobertura vacinal não foi universal; } \\
\text { abordagens educativas são necessárias } \\
\text { para esclarecer controvérsias sobre a } \\
\text { eficácia, eventos adversos e benefícios }\end{array}$ \\
\hline
\end{tabular}




\begin{tabular}{|c|c|c|c|}
\hline $\begin{array}{|lr|}\text { estudo } & \text { transversal } \\
\text { de } & \text { base } \\
\text { populacional. }\end{array}$ & & $\begin{array}{l}\text { associados à vacinação } \\
\text { em idosos, identificar } \\
\text { motivos da não adesão } \\
\text { e eventos adversos } \\
\text { pós-vacinação. }\end{array}$ & da vacinação contra influenza. \\
\hline \begin{tabular}{|l|} 
Fatores \\
associados \\
aceitação à \\
vacina influenza \\
entre \\
trabalhadores de \\
saúde: \\
conhecimento, \\
atitude e prática. \\
\end{tabular} & $\begin{array}{l}\text { SOUZA, T. } \\
\text { LOBÃO, W. M; } \\
\text { SANTOS, C. A } \\
\text { S. T; ALMEIDA, } \\
\text { M. C; JÚNIOR, } \\
\text { E. M. }\end{array}$ & $\begin{array}{l}\text { Identificar } \\
\text { associados } \\
\text { vacinação atores } \\
\text { influenza, contra } \\
\text { com trabalhadores de } \\
\text { saúde de um grande } \\
\text { Complexo Hospitalar de } \\
\text { Salvador, Bahia. }\end{array}$ & $\begin{array}{l}\text { A cobertura da vacina influenza foi de } \\
61,5 \% \text {, sendo maior entre enfermeiros } \\
(69,0 \%) \text { e menor entre médicos }(49,1 \%) \\
\text { Os fatores associados a vacinar-se } \\
\text { contra influenza foram: conhecer que } \\
\text { mesmo estando saudável deve- se } \\
\text { vacinar; saber que a vacina não protege } \\
\text { por muitos anos; e não temer efeitos } \\
\text { adversos pós- vacinais. }\end{array}$ \\
\hline $\mid \begin{array}{lr}\text { Motivos da } & \text { não } \\
\text { adesão } & \text { de } \\
\text { crianças } & \text { à } \\
\text { campanha } & \text { de } \\
\text { vacinação contra a } \\
\text { influenza. }\end{array}$ & $\begin{array}{l}\text { SIEWERT, J. S; } \\
\text { CLOCK, } \\
\text { MERGNER, } \\
\text { G; ROCHA, P. F. } \\
\text { A; ROCHA, M. } \\
\text { D. H. } \\
\text { ALVAREZ, A. M. }\end{array}$ & $\begin{array}{l}\text { Conhecer os motivos } \\
\text { da não adesão dos } \\
\text { pais/responsáveis de } \\
\text { crianças à campanha } \\
\text { de vacinação contra a } \\
\text { influenza. }\end{array}$ & $\begin{array}{l}\text { A enfermagem tem papel fundamental } \\
\text { na elaboração, planejamento e execução } \\
\text { das campanhas de vacinação. Este } \\
\text { estudo evidenciou a necessidade de } \\
\text { melhorar as estratégias de educação em } \\
\text { saúde referentes à vacinação contra o } \\
\text { vírus da influenza e ampliar o acesso na } \\
\text { Atenção Primária. }\end{array}$ \\
\hline 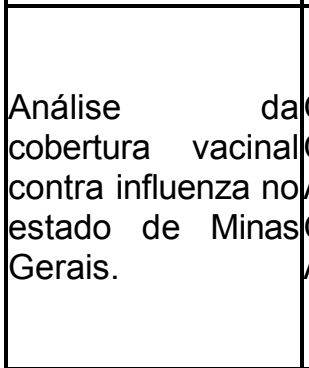 & $\begin{array}{l}\text { GONTIJO, T. L.; } \\
\text { OLIVEIRA, V. C.; } \\
\text { ALMEIDA, N. A.; } \\
\text { GUIMARÃES, E. } \\
\text { A. A. }\end{array}$ & $\begin{array}{l}\text { Analisar a cobertura } \\
\text { vacinal contra influenza } \\
\text { de idosos, gestantes e } \\
\text { crianças menores de } \\
\text { dois anos nos } 853 \\
\text { municípios do estado } \\
\text { de Minas Gerais. }\end{array}$ & $\begin{array}{l}\text { O estado de Minas Gerais atingiu a } \\
\text { cobertura vacinal contra influenza, porém } \\
\text { percebe-se baixa homogeneidade da } \\
\text { cobertura no grupo gestante e crianças. } \\
\text { Mesmo havendo sucesso na campanha } \\
\text { vacinal é necessário compreender } \\
\text { melhor os fatores que expliquem baixas } \\
\text { coberturas em alguns municípios para } \\
\text { auxiliá- los no alcance de suas metas. }\end{array}$ \\
\hline 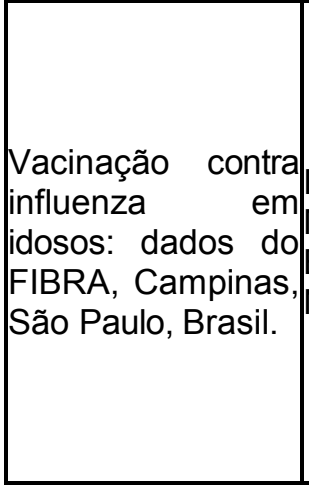 & $\begin{array}{l}\text { FRANCISCO, } \\
\text { M. S. S. } \\
\text { BORIM, F. S. A } \\
\text { NERI, A. L. }\end{array}$ & $\begin{array}{l}\text { Verificar a prevalência } \\
\text { de vacinação contra } \\
\text { Influenza, segundo } \\
\text { indicadores rede de } \\
\text { capacidade funcional, } \\
\text { fragilidade, suporte e } \\
\text { envolvimento social e } \\
\text { estado de saúde, com } \\
\text { informações obtidas } \\
\text { por meio de inquérito } \\
\text { específico para a } \\
\text { população idosa. }\end{array}$ & \begin{tabular}{|l|} 
Dos 679 idosos, $74,4 \%$ referiram \\
vacinação no ano prévio. A prevalência \\
de vacinação foi significativamente maior \\
entre os homens e menor naqueles com \\
maior escolaridade. A lentidão de marcha \\
associou-se positivamente à vacinação, \\
assim como a maioria dos indicadores \\
de envolvimento social.
\end{tabular} \\
\hline
\end{tabular}

Quadro 1: Identificação dos artigos utilizados na pesquisa. Fonte: Dados da pesquisa, 2019.

Algumas pessoas, como idosos, crianças, gestantes e pessoas com alguma comorbidade, possuem um risco maior de desenvolver complicações devido à influenza. A melhor maneira de se prevenir contra a doença, é vacinar-se anualmente. A vacina é capaz de promover imunidade durante o período de maior circulação dos vírus influenza reduzindo o agravamento da doença. A estratégia de 
vacinação na rede pública de saúde foi sendo ampliada e, atualmente, a vacinação é indicada para indivíduos com 60 anos ou mais de idade, população privada de liberdade e funcionários do sistema prisional, povos indígenas, crianças com idade de 6 meses a menor de 5 anos, profissionais de saúde, pessoas portadoras de doenças crônicas não transmissíveis e outras condições clínicas especiais (conforme listagem definida pelo Ministério da Saúde com sociedades científicas), gestantes e puérperas (BRASIL, 2015).

Em complemento da explanação acerca da efetividade de vacina contra a influenza, Bacurau (2019) observa que houve uma redução nos coeficientes de mortalidade por doenças cerebrovasculares no período após o início das campanhas de vacinação.

Ao estudar a adesão da vacinação contra a gripe em idosos, Moura (2015) observou 1.399 idosos que representaram cerca de 1.017.588 idosos do Município de São Paulo. Foi evidenciado que a cobertura vacinal atingiu cerca de $73,8 \%$ e que, dos idosos não vacinados, $68 \%$ não souberam informar ou não responderam o motivo da não adesão, porém, o principal motivo apresentado por $8,3 \%$ dos idosos foi a de não acreditar na vacina.

Em seu estudo, Bacurau (2018) identificou uma baixa prevalência de vacinação em adultos e idosos portadores de Doenças Respiratórias Pulmonares Crônicas (DRPC) ao estudar a vacinação contra a gripe em pacientes portadores de DRPC utilizando-se de dados da Pesquisa Nacional de Acesso, Utilização e Promoção do Uso Racional de Medicamentos (PNAUM) cota 2013-2014.

Francisco (2015), ao estudar a prevalência de vacinação contra influenza em idosos, percebeu instituições de promoção de saúde e de prevenção de agravos necessitam ser mais reconhecidas pelos idosos e pelos profissionais de saúde. Além disso, observou-se que os profissionais de saúde, de serviços privados ou públicos, precisam sensibilizar os idosos quanto adesão da vacina.

Não obstante, Neves (2016) observou que o sexo não se mostrou associado à realização da vacinação ainda que o sexo feminino tenha mais utilização dos serviços de saúde, assim como também de informação acerca das doenças. Também foi reforçado que a prática de atividade física no lazer se mostrou 
associada à vacinação e que, pessoas com estilo de vida mais saudável se previnam mais e, por consequência, se vacinem mais.

Em se tratando de profissionais da saúde, Souza (2019) aponta que os enfermeiros foram os trabalhadores que mais se vacinaram e, de contrapartida, a cobertura mais baixa ocorreu entre os profissionais médicos. Ainda, houve uma associação significativa entre não ter medo das reações adversas pós-vacinais e aceitar se vacinar contra a influenza.

Mizuta (2019) identificou que embora a maioria dos médicos e estudantes reconheça que os indivíduos vacinados podem proteger outras pessoas da aquisição de doenças infecciosas, dos 92 alunos do curso de medicina da Faculdade de Medicina e Odontologia São Leopoldo Mandic, Campinas São Paulo) estudados, cerca de $34 \%$ não reconheceram a importância da proteção de rebanho no controle de doenças infecciosas.

$\mathrm{Na}$ população infantil, Costa (2019) aponta que há poucas evidências a respeito da percepção dos pais sobre a vacinação contra a influenza em crianças sadias que frequentam creches e que os fatores que aumentam adesão à vacinação contra a influenza é a recomendação da mesma por um profissional médico e a percepção de que a criança está em risco de adquirir a doença.

Ainda, Gontijo (2017) em seu estudo com municípios do estado de Minas Gerais, verificou que a cobertura vacinal em idosos vem apresentando crescimento, todavia o grupo de gestante apresentou maior dificuldade no alcance da cobertura vacinal, justificada em fatores como: valores pessoais e familiares, situação socioeconômica desfavorável, consultas de pré-natal inadequadas, falta de material e infraestrutura, dentre outros.

\section{CONCLUSÃO}

Como a forma mais eficaz de evitar a infecção influenza, a imunização tem sido uma peça-chave na saúde preventiva do Brasil, e verifica-se grande progresso da cobertura vacinal da população. Todavia, ainda se tem como desafio desconstruir 
a cultura da anti-vacinação, bem como minimizar as motivações para não adesão à vacinação e investir em estudos que contribuam para a atuação efetiva do Programa Nacional de Imunizações (PNI).

A revisão sobre a adesão à vacina contra a influenza permitiu observar publicações muito relevantes sobre a questão. Nessa perspectiva, evidenciou-se que a maioria dos estudos são voltados para os idosos, o que confirma a necessidade de que sejam desenvolvidos novos estudos que abranjam os demais grupos subsidiados pela rede pública de saúde, sendo eles: população privada de liberdade e funcionários do sistema prisional, povos indígenas, pessoas portadoras de doenças crônicas não transmissíveis e outras condições clínicas especiais, crianças com idade de 6 meses a menor de 5 anos e profissionais de saúde, gestantes e puérperas.

Ainda, se faz mister destacar a escassez de estudos e pesquisas relacionados à adesão da vacina influenza entre crianças e adolescentes, assim como também acerca das variáveis que interferem diretamente neste processo. 


\section{REFERÊNCIAS BIBLIOGRÁFICAS}

BACURAU, A. G. M; FERRAZ, R. O; DONALISIO, M. R; FRANCISCO, P. M. S. B.Mortalidade por doenças cerebrovasculares em idosos e a vacinação contra a influenza: Estado de São Paulo, Brasil, 1980-2012. Cadernos de Saúde Pública. v.35, n.2, p.1-14, 2019.

BACURAU, A. G. M; FRANCISCO, P. M. S. B. Prevalência de vacinação contra gripe nas populações adulta e idosa com doença respiratória pulmonar crônica. Cadernos de Saúde Pública. v.34, n.5, p.1-6, 2018.

BRASIL. 19a Campanha Nacional de Vacinação Contra a Influenza: informe técnico. Coordenação Geral do Programa Nacional de Imunizações. Departamento de Vigilância Epidemiológica, Secretaria de Vigilância em Saúde, Ministério da Saúde, 19ª Ed. Brasília, 2017.

BRASIL. Protocolo de Tratamento da Influenza. Ministério da Saúde. Secretaria de Vigilância em Saúde. Departamento de Vigilância das Doenças Transmissíveis. Brasília-DF, 2015.

COSTA, P; MENESES, N. F. A; ANDRADE, P. R; HINO, P; TAMINATO, M. Adesão à Vacinação Contra Influenza. Revista de Enfermagem UFPE On Line. v.13, n.14, p.11511156, 2019.

FRANCISCO, P. M. S. B.; BORIM, F. S. A.; NERI, A. L. Vacinação contra influenza em idosos: dados do FIBRA, Campinas, São Paulo, Brasil. Ciência e Saúde Coletiva. v.20, n.12, p.37753786, 2015.

GONTIJO, T. L.; OLIVEIRA, V. C.; ALMEIDA, N. A.; GUIMARÃES, E. A. A. Análise da cobertura vacinal contra influenza no estado de Minas Gerais. Revista Ciência Enfermagem. v.23, n.3, p.69-75, 2017.

MIZUTA, A. H; SUCCI, G. M; MONTALLI, V. A. M; SUCCI, R. C. M. Percepções Acerca da Importância das Vacinas e da Recusa Vacinal numa Escola de Medicina. Revista Paulista de Pediatria. v.37, n.1, p.34-40, 2019.

MOURA, R. F; ANDRADE, F. B; DUARTE, Y. A.O; LEBRÃO, M. L; ANTUNES, J. L. F. Fatores associados à adesão à vacinação anti-influenza em idosos não institucionalizados, São Paulo, Brasil. Cadernos de Saúde Pública. v.31, n.10, p.2157-2168, 2015.

NEVES, R. G; DURO, S. M. S; TOMASI, L. Vacinação contra influenza em idosos de PelotasRS, 2014: um estudo transversal de base populacional. Revista Epidemiologia e Serviços de Saúde. v.25, n.4, p.755-766, 2016.

OMS. Organização Mundial da Saúde. 2017. Disponível em: https://www.paho.org/bra/index.php?option=com_content\&view=article\&id=5429:opasomsincentiva-publico-alvo-a-se-vacinar-contra-a-gripe-no-brasil\&ltemid=820. Acesso em: 03/01/2020.

SIEWERT, J. S; CLOCK, D; MERGNER, P. G; ROCHA, P. F. A; ROCHA, M. D. H. A; ALVAREZ, A. M. Motivos da Não Adesão de Crianças à Campanha de Vacinação Contra a Influenza. Revista Cogitare Enfermagem. v.23, n.3, p.1-8, 2018.

SOUZA, T. P; LOBÃO, W. M; SANTOS, C. A. S. T; ALMEIDA, M. C. C; JÚNIOR, E. D. M. Fatores Associados à Aceitação da Vacina Influenza entre Trabalhadores de Saúde: Conhecimento, Atitude e Prática. Ciência e Saúde Coletiva. v.24, n.8, p.3147-3158, 2019. 